\title{
A Practical Guide to Measuring Social Structure Using Indirectly Observed Network Data
}

\author{
TYLER H. MCCORMICK, ${ }^{1}$ AMAL MOUSSA, ${ }^{2}$ JOHANNES \\ RUF, ${ }^{3}$ THOMAS A. DIPRETE, ${ }^{4}$ ANDREW GELMAN, ${ }^{5}$ \\ JULIEN TEITLER, ${ }^{6}$ AND TIAN ZHENG ${ }^{7}$ \\ ${ }^{1}$ Department of Statistics and Department of Sociology, University of \\ Washington, Seattle, Washington, USA \\ ${ }^{2}$ Unaffiliated \\ ${ }^{3}$ University of Oxford, Oxford-Man Institute of Quantitative Finance and \\ Mathematical Institute, Oxford, United Kingdom \\ ${ }^{4}$ Department of Sociology, University of Wisconsin-Madison, Madison, \\ Wisconsin, USA \\ ${ }^{5}$ Department of Statistics and Department of Political Science, Columbia \\ University, New York, New York, USA \\ ${ }^{6}$ School of Social Work, Columbia University, New York, New York, USA \\ ${ }^{7}$ Department of Statistics, Columbia University, New York, New York, USA
}

\begin{abstract}
Aggregated relational data (ARD) are an increasingly common tool for learning about social networks through standard surveys. Recent statistical advances present social scientists with new options for analyzing such data. In this article, we propose guidelines for learning about various network processes using ARD and a template to aid practitioners. We first propose that ARD can be used to measure "social distance" between a respondent and a subpopulation (individuals named Kevin, those in prison, or those serving in the military). We then present common methods for analyzing these data and associate each of these methods with a specific way of measuring social distance, thus associating statistical tools with their underlying social science phenomena. We examine the implications of using each of these social distance measures using an Internet survey about contemporary political issues.
\end{abstract}

AMS classifications: 62-07; 62P25; 91D30.

Keywords: Aggregated relational data; hierarchical model; opinion formation; sampling bias; overdispersed Poisson distribution; sample survey; social network.

\section{Introduction}

Though the overwhelming majority of methods for analyzing network data assume complete network data are available, these data are financially or logistically impossible to collect, an issue Burt (1982) calls "the single factor most restricting structural theory."

Received January 2012; accepted November 2012.

The first three authors contributed equally to this paper.

Address correspondence to: Tian Zheng, Columbia University, School of Social Work, 1255 Amsterdam Avenue, New York, NY 10027, USA. Email: tzheng@ stat.columbia.edu 
Aggregated relational data (ARD) are one increasingly popular alternative for measuring networks indirectly. ARD, introduced by Killworth et al. (1998a), are answers to questions of the form "How many X's do you know?" where "X" represents a subpopulation of interest. Thus, instead of measuring direct relationships between actors as in the complete network case, we observe the frequency with which an actor interacts with a particular group. ARD are often used to predict characteristics of populations that are difficult to reach using standard surveys (Killworth et al., 1998b) and more recently to learn about polarization and segregation (DiPrete et al., 2010). These data do not require any specific sampling technique and are easily integrated into standard surveys.

Since we measure network features indirectly, the standard network statistics for complete network data are no longer quantifiable. New statistical methods have been developed for describing network features from these data, though little has been done to explore their interpretation. We do this by conceptualizing ARD-based statistics as a measure of social distance. Specifically, we compare two ways of measuring social distance, which we refer to as counts and residuals. By counts we mean the raw responses to our ARD questions, which are currently the most common way in which indirect network measures are included in analysis. The counts, which represent the frequency of interactions between an actor and members of a given subpopulation, are a natural starting point for measuring social distance and are represented directly by ARD. Actors who interact more frequently with a subpopulation should, intuitively, be more socially proximate than those who interact infrequently. A social scientist who includes an ARD count as a covariate in a regression model, therefore, adjusts for the level of exposure to the subpopulation of interest.

We contrast the raw counts with residuals from an overdispersed Poisson regression model based on Zheng et al. (2006) The Zheng et al. (2006) model adjusts for respondent degree, or total network size, and the size of the subpopulation. Using the residuals from this model yields an ARD-based statistic that measures the connectivity of the respondent to a given subpopulation in excess of what would be expected for a person with a similar network size. For two respondents who report knowing the same number of people on welfare, for example, we would conclude that the respondent with an acquaintanceship network of size 500 would be socially closer to the welfare population than a respondent with an 800-person network.

In the remainder of this section we discuss the motivation for measuring network features using counts and residuals. In section 2 we describe the data we used to develop this method and the overdispersed Poisson regression model, and we define the residuals which we consider as one possible measure of social structure. We present results regarding social structure and opinion formation in section 3 . We use these distinctions to suggest guidelines for data analysis. Section 4 discusses the main findings of our work.

\subsection{Motivation for Measuring Counts and Residuals}

The motivation for the two measures we consider comes from the scientific context of our data. Specifically, we wish to measure social structure through indirect observations of respondents' social networks. Counts and residuals are found to reveal different aspects of social structure. A pertinent question for social science researchers is then which of the two to include in an analysis. We compare the performance of the two measures as indicators of social distance, specifically as predictors of respondent opinions regarding contemporary political and social issues. 
One possible interpretation of social distance is related to influence. Burt (1987), for example, defines equivalence as similarity in the pattern of two actors' network relations. Two actors are proximate if they interact with the network in similar ways (have the same friends, for example). Without information about specific members of the network, as in the ARD case, we can conceive both the counts and residuals as a form of equivalence, which we term weak equivalence. The key distinction in our definition is that we define social distance in terms of the expected social distance between a respondent and an entire group of actors, rather than any specific actor.

\section{Data and Model}

In this section we describe the data set, the overdispersed Poisson regression model, and potential measures of social distance.

\subsection{Data}

We developed this method on data from the Cooperative Congressional Election Study (CCES), a large national online survey created by 30 universities (MIT Web 2007). Each university has created a module of about 120 questions for 1000 respondents. The survey was conducted by Polimetrix in October and November 2006. For each survey of 1000 persons, half of the questionnaire is developed by an individual research team, and half of the questionnaire is given by common content (MIT Web 2007), which consists of approximately 60 questions, 40 in the preelection wave about general political attitudes, various demographic factors, voting choices, and political information, and 20 in the postelection wave. In addition to these questions, Polimetrix provides demographic indicators, party identification, ideology, and validated votes obtained after the 2006 election. Our data set comes from Columbia University's module.

Polimetrix uses a random sample matching methodology to produce "representative" samples from nonrandomly selected samples of respondents: First a target random sample is drawn from the U.S. population, and then each member of the target sample is matched with a respondent by minimizing a distance function on a large set of variables so that the respondent is as similar as possible to the selected member of the target sample. Thus, the matched sample has characteristics similar to those of the target sample.

Using an Internet survey can be a problem for generating a representative sample; there tend to be fewer elderly Internet users than young Internet users, yet among the Internet users the propensity for participation in survey research is higher for elderly users than for young users (MIT Web 2007). Thus, there is a preselection effect that can generate a misrepresentative sample where certain groups are underrepresented. The survey could be also biased toward politically active people, since $89 \%$ of the respondents claim to have voted in the 2006 elections, while the overall voter turnout was substantially lower.

Respondents were asked various questions related to their socioeconomic and personal characteristics (e.g., race, gender, education, income), to their political opinions (e.g., approval of a timetable in Iraq, approval of George W. Bush's handling of Iraq), and to their social network (e.g., how many Kevins or Brendas they know, how many unemployed persons and people on welfare they know). "To know" in our study is defined as knowing a person's name and being willing to stop and to talk to that person for at least a moment. We have ARD for $K=13$ subpopulations and $n=994$ respondents (six of the original 1000 respondents did not answer any of the questions). Respondents were presented with 
five possible choices: $0,1,2$ to 5,6 to 10 , and more than 10 persons, which constitutes an interval-based data set.

\subsection{Model}

The classical Erdös-Renyi model for social links, which assumes that links between people in the population are formed completely randomly (i.e., the probability that two persons get to know each other is the same whoever those persons are), implies that the number $v_{i, k}$ of persons in subpopulation $k$ that respondent $i$ knows follows a Poisson distribution with intensity $\bar{a} b_{k}$, where $\bar{a}$ is the expected network size of a randomly selected member of the population and $b_{k}$ is the expected number of links involving subpopulation $k$ divided by the total expected number of social links (popularity of subpopulation $k$ ).

In this model, all individuals have the same expected degree. This is clearly not the case in the population at large. Some individuals are in more socially exposed positions (members of the clergy or politicians, for example) and there is natural variability in gregariousness. Furthermore, numerous previous studies using ARD have also found variation in excess of what would be expected under a Poisson model (Newman 2003). This superPoisson variation is known as overdispersion and results from the propensity for individuals to know either no members of a subpopulation or multiple members. To illustrate, the answers for "How many Rachels do you know?" and "How many people on welfare do you know?" are summarized in Figure 1. The histograms show an overdispersion for the distributions of both groups, meaning that most people know few Rachels and few people on welfare, but some people know many Rachels and many people on welfare, with a more pronounced overdispersion for the "Welfare" group than for the "Rachel" group.

To address these two issues, Zheng et al. (2006) propose an overdispersed model where individual $i$ has also an individualized propensity $g_{i, k}$ to know people from the subpopulation $k$, formally: $v_{i, k} \sim$ Poisson $\left(a_{i} b_{k} g_{i, k}\right)$ where $a_{i}$ is the degree (network size) for respondent $i$. Zheng et al. (2006) assume this propensity $g_{i, k}$ follows a gamma distribution with mean 1 and shape parameter $1 /\left(\omega_{k}-1\right)$, where $\omega_{k}$ is the overdispersion parameter,
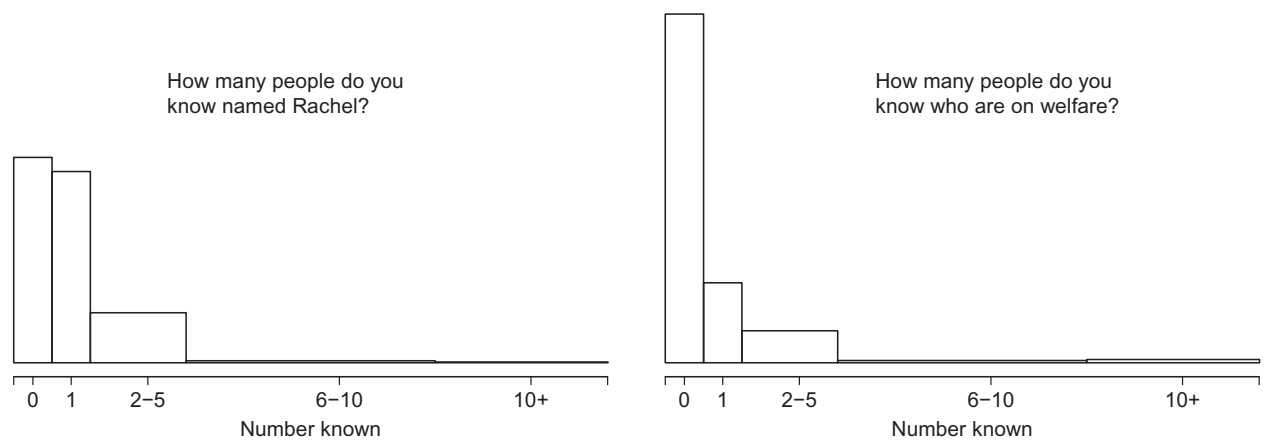

Figure 1. Histograms showing the distribution of the answers of two typical questions: "How many Rachels do you know?" and "How many people on welfare do you know?" The counts are represented by the area (not the height) of each of the bars. The heights of the last two bins on the left histogram correspond to 23 and 8 respondents, on the right histogram to 27 in both bins. The larger ratio of the heights of the first and second bar in the right histogram compared to the left one indicates a higher overdispersion in the "Welfare" group. The estimated overdispersion parameters $\omega$ are here 1.2 for the Rachel group and 5.4 for the "Welfare" group. 
whose simplest interpretation is a scale of the variance: $\operatorname{Var}\left(v_{i, k}\right)=\omega_{k} E\left(v_{i, k}\right)$. The overdispersion accounts for the extra variance of the data. The probability distribution of $v_{i, k}$ is negative binomial with mean $a_{i} b_{k}$ and overdispersion parameter $\omega_{k}$.

We refer readers to Zheng et al. (2006) for details about the model and priors and to Appendix A in DiPrete et al. (2010) for how to adapt this model for interval data. Additionally, McCormick et al. (2009) provide a description of the starting values and further implementation details, including subpopulation sizes needed for normalization.

\subsection{Measures of Social Distance}

A simple measure of social distance is the count, $y_{i, k}$, which measures the number of people known by person $i$ in group $k$. Since the responses are in intervals, we define counts based on the midpoints of the interval as

$$
y_{i, k}:=\mathbf{1}_{\left\{v_{i, k}=1\right\}}+3.5 * \mathbf{1}_{\left\{2 \leq v_{i, k} \leq 5\right\}}+8 * \mathbf{1}_{\left\{6 \leq v_{i, k} \leq 10\right\}}+15 * \mathbf{1}_{\left\{10<v_{i, k}\right\}} .
$$

We map each interval on its midpoint and interpret this point as the number of people, which a certain respondent knows in a subpopulation. For the largest interval, namely more than 10 people, we assign the value 15 .

As an alternative measure of social distance, we define the residuals as the difference between the square root of the observed counts and their expectation from the model in section 2.2,

$$
r_{i, k}:=\sqrt{y_{i, k}}-E\left(\sqrt{Y_{i, k}}\right),
$$

where the square root is introduced to stabilize the variance. A small residual means that a respondent knows about as many people in a certain subpopulation as would be expected from her or his network size.

In summary, the count $y_{i, k}$ represents a combination of respondent $i$ 's exposure to and level of knowledge of subpopulation $k$. Therefore, counts depend not only on the social network structure but also on the respondent's network size. In contrast, the residuals represent connectedness with the subpopulation as deviations from the expected. Thus, they indicate social structure more directly because they measure connectedness with a subpopulation net of what would be expected simply from the size of the subpopulation and the respondent's network size.

\section{Results}

In the previous section we presented two candidate methods of measuring social distance using indirectly observed network data, counts, and residuals. Here, we explore the different types of information about the network given by each of these methods. We contend that, in accounting for degree, the residuals measure the respondent's average social distance to members of the subpopulation. Without adjusting for degree, the counts measure directly the frequency of interaction between a respondent and members of a subpopulation. We then examine these measures as predictors of opinions, and in doing so further explore the impact of social distance on opinion formation. 
We analyze the information about social structure in counts and residuals in section 3.1 and compare their predictive power on opinion formation in section 3.2. Last, we discuss in section 3.3 a way to detect sampling bias in a survey relying on ARD questions.

\subsection{Measuring Social Structure With ARD}

Using ARD, we observe only the aggregated number of ties between a respondent and a particular subpopulation. The indirect nature of our data makes the standard networkbased measures of social structure inapplicable. Instead, we compare two measures based on ARD. Our raw counts reflect the number of interactions between a respondent and the subpopulation of interest. In general, we posit that respondents with a higher frequency of interaction with a subpopulation are more proximate. While this does not require any additional modeling, the raw counts do not account for the total volume of a respondent's ties. In contrast, residuals as defined in section 2.3 adjust for the respondent's network size and the relative size of the subpopulation. The remaining information represents the tendency for a respondent to know someone in the subpopulation in excess of what would be expected for someone with their network size. We contend that the model residuals more reasonably represent social structure than the raw counts, which do not separate the structure of overdispersion from the degree distribution and relative size of the subpopulation in question. Instead, the raw counts indicate a more coarse level of knowledge of, or exposure to, the subpopulation.

To understand the information contained in these measures, we first analyze patterns in the measures across the 13 subpopulations. We consider hierarchical clustering (see, e.g., Venables and Ripley 2002) using Kendall's tau as a distance measure. We apply the clustering algorithm to both the residuals and the counts and consider both the final pairing and the levels at which particular subpopulations break in the dendrograms as evidence in similarity in profiles. Subpopulations breaking at lower levels of the tree, for example, are considered more similar. After standardizing the counts and residuals, we also apply multidimensional scaling (see Hastie et al. 2001). We use two dimensions for an easily interpretable visual display of the similarity between profiles for the subpopulations.

Figure 2 shows multidimensional scaling and hierarchical clustering based on the (standardized) residuals and counts of number known in each of the subpopulations. Though the primary comparison for the multidimensional scaling plots is still within each graph, we use a common center and rotation to facilitate comparison of the general pattern across graphs.

In Figure 2, the names are also more widely spaced for the residuals than for the counts. The similarity of the position of the names within each graph is a bit misleading. Aside from the male names being closer to the male names and female names being closer to other female names, there is little reason to believe that the names should be socially close. This result is consistent, however, with the finding of Zheng et al. (2006) that the residuals for the names are slightly correlated. One possible explanation is that some people remember names better than events. Nonetheless, the six names are nearly on top of one another for the counts. For the residuals, the names are still close together, but noticeably less than for the counts. The counts are confounded by degree, or network size, and thus display less resolution than the residuals. The distance between the names in the residual plot (right) is more reasonable, where the "military" and "Iraq" populations are closer together than "Keith" to "Kevin," for example. Similarly, the names are farther away from one another and from the subpopulations on the dendrogram for the residuals, whereas their position is more similar to the subpopulations on the dendrogram for the counts. 


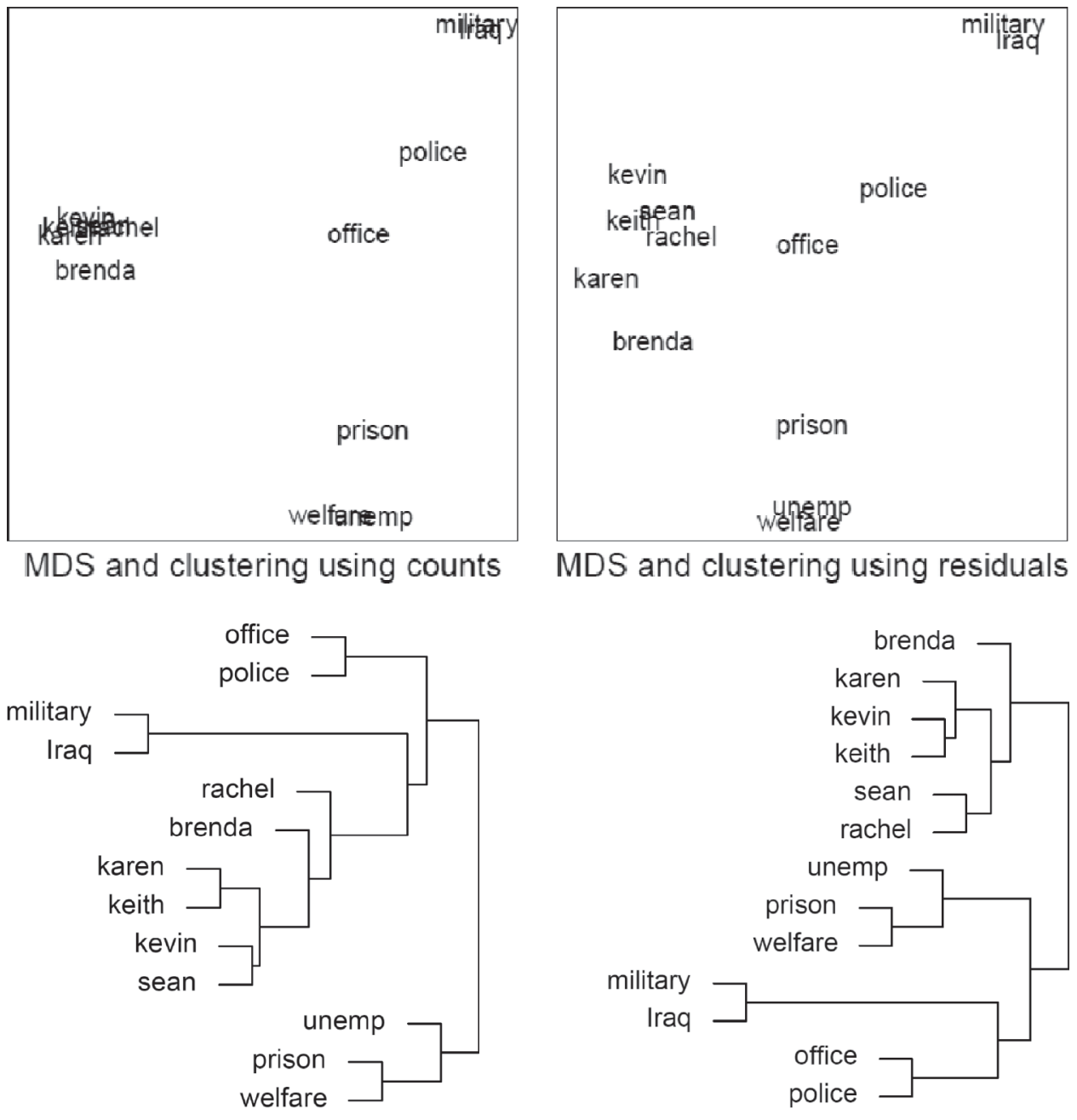

Figure 2. Hierarchical clustering and multidimensional scaling (MDS) using raw counts and model residuals. For ease of comparison, we use a common orientation rotated based on the first names and centered using the number known who have run for office. The spacing of the names is more appropriate when using residuals than counts, indicating that the information about social structure contained in the counts is confounded with degree.

The distinction between the left and right panels of Figure 2 indicates that the counts and residuals convey different information about the responses. In controlling for degree, the right plot of Figure 2 represents additional structure once total network volume has been accounted for. In the counts, much of the information about social structure is masked by degree. There is, in essence, no way of knowing whether a respondent who reports knowing a large number of members of a subpopulation could be proximate to the subpopulation, or could simply have a very large network. The counts, therefore, represent a respondent's exposure, or level of knowledge, of the subpopulation group. In contrast, the residuals represent connectedness with the subpopulation in excess of the expected, which indicates social structure more directly. 


\subsection{Case Study: Social Structure and Political Ideology}

We now demonstrate the use of ARD-based statistics for the type of analysis we believe would be common for many social science researchers. Our goal in this section is not to make substantive claims in our application area, but rather to evaluate the implications of the distinct information the two quantities provide. As an example, we consider the association between social structure and political opinions. Opinion formation, as with influence processes in general, relies on a highly dependent series of interactions between connected actors and is often confounded by selection. Exactly how this influence manifests as a change in opinion, the so-called substantive nature of influence (Burt 1987), is a challenging problem.

Both counts and residuals measure a generalized form of similarity based on the types of people with which a respondent interacts and measured by their social distance. In the context of marriage, for instance, Kalmijn (1994) contends that the cultural aspects of social status are more influential than economic aspects in the assortative matching process. That is, an individual who is culturally more similar to members of a higher social class is a more valuable partner than one who is of a higher economic class. In many cases we also have information about a respondent's membership in a particular group (attending church, serving in the military, for example). Membership status provides a more direct measure of similarity (people who attend church are united through common beliefs, for example). We begin by exploring the association between belonging to a particular group and respondents' opinions. We then compare the magnitude of these associations with the more general form of similarity measured through ARD.

Figure 3 presents coefficients and standard errors for regression models predicting opinions. For each of the subpopulations (people on welfare, in prison, etc.), the respondent's opinion (approving of Bush's handling of Iraq, supporting late-term abortion, etc.) was regressed once on the counts and once on the residuals. We used logistic regression for opinions with dichotomous responses (support, oppose) and proportional-odds logistic regression (see, e.g., Venables and Repley 2002) for questions where respondents answered on a scale (support, neutral, oppose). In each model, we controlled for the respondent's political ideology, political party, and demographic characteristics (age, gender, race, income, education, employment status).

We consider first the regressions using group membership as a covariate rather than ARD. We find a significant negative relationship between the frequency of church attendance and being in favor of late abortion (standardized coefficient -.86 and standard error .16). Those who attend church regularly were more likely to hold views consistent with major religious groups. Regressing being in favor of federal funding for stem cell research on the frequency of church attendance indicates that frequent churchgoers were less likely to be in favor of stem cell research (standardized coefficient -.97 and standard error .17). Also, knowing that an individual is serving in the military or has immediate family members in the military, for example, establishes that many of the respondent's closest contacts are also members of the service. Having immediate family members currently serving in the military has a positive influence on approving Bush's handling of Iraq (standardized coefficient .60 and standard error .30), for example. These results are consistent with the work of Erickson (1988) and the preceding work of Moscovici (1985), which suggest that an individual's decisions are guided by their comparison to groups of similar peers.

We now turn our attention to our two measures of social distance, counts and residuals. Using both measures, we found evidence of a relationship between social distance and 


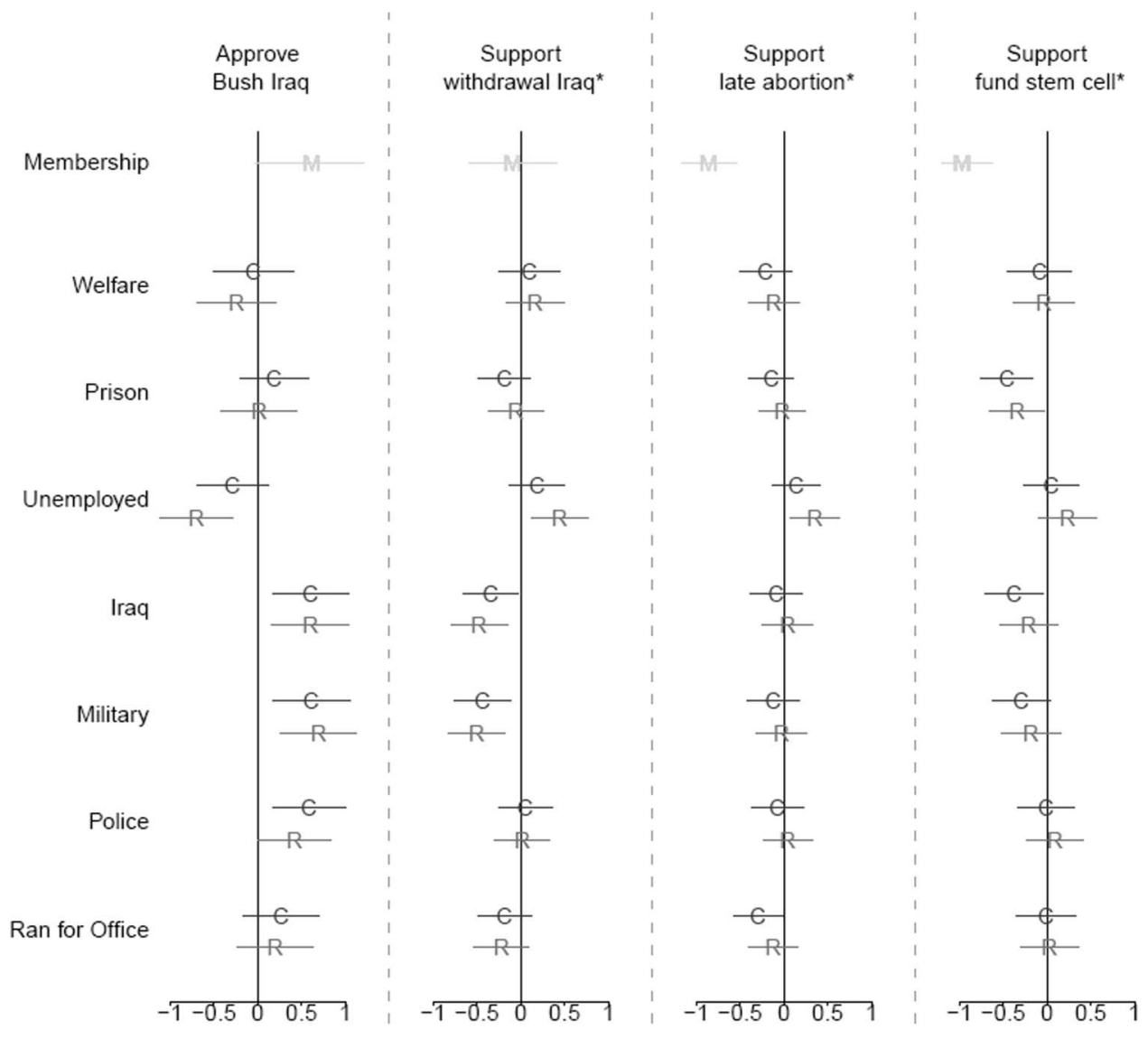

Figure 3. Coefficients and standard errors for counts, residuals, and memberships. Each point represents a separate regression of the respondent's opinion (controlling for political and demographic factors) on either counts, residuals or membership. There are in total 60 regressions. " $\mathrm{C}$ " is a coefficient for counts, " $R$ " for residuals, and " $M$ " for membership. Membership for the Iraq questions means that the respondent or an immediate family member is an active member of the military. For the remaining two questions a respondent was considered a member if the respondent attends church regularly. For the three questions with a star, respondents could answer in the affirmative, the negative, or say "not sure." For the remaining question, respondents could answer either in the affirmative or in the negative. Overall the signal is most pronounced for the military and unemployed subpopulations. In both cases the coefficients for residuals tend to be more extreme than those for the counts.

respondent opinion. For example, being more socially proximate to those serving in the military was associated with approving of George W. Bush's handling of the war in Iraq (standardized coefficient for residuals .70 and standard error .22 and for counts .61 with standard error .22) and being more likely to support withdrawal from Iraq (standardized coefficient for residuals -.50 and standard error .16 and for counts -.43 with standard error .16). Though the differences are not statistically significant, the magnitudes for military-related opinions were smaller for those socially close to individuals serving in Iraq than for those close to the military in general (e.g., standardized coefficient for approving of Bush was, using residuals, .60 with standard error .22 and with counts a coefficient of .60 and standard error .22 for Iraq). We posit that the counts 
are confounded by the respondent's degree. With counts, an individual who has a high degree and high number of associations in the military receives the same social distance as someone who has a very small degree and a high number of associations. We do not expect degree to be associated with an individual's opinion, and thus we would expect the opinions of the individual with a larger degree to be less homogeneous, which would result in coefficients of smaller magnitude. In other words, people with high residuals who support a position are socially close to the subpopulation in question. Individuals with high counts may be socially close but they may also simply have a high degree.

We found similar patterns in counts and residuals when measuring social distance to the unemployed, even though the signal direction is nearly the exact opposite of military. We found that those socially close to the unemployed were less likely to support George W. Bush's plan for Iraq (standardized coefficient for residuals -.70 and standard error .21 and for counts -.29 with standard error .20) and more willing to support abortion rights (standardized coefficient for residuals .35 and standard error .14 and for counts .14 with standard error .14).

\subsection{Latent Sampling Bias}

Despite the overlap in the subpopulations, the signal is much weaker for the welfare group than for the unemployed. Even more surprising is that the coefficients for counts in the prison subpopulation are typically more extreme than those of the residuals. We believe that the weaker signal in these subpopulations could be partially attributable to network-based sampling bias, which we now describe.

Since this is an Internet survey, there were additional efforts to ensure a representative sample, as discussed in section 2.1. Despite these efforts, we found that individuals who are socially close to individuals in prison or on welfare were underrepresented. This fact is perhaps not surprising since members of both of these subpopulations are often impoverished and thus they, or individuals they are socially close to, may have difficulty accessing the Internet. These observations reveal a latent bias in the sampling procedure of this internet survey. Figure 4 displays the actual fractional subpopulation size (see the Appendix of McCormick et al. 2009) against the fractional subpopulation size estimated by the Zheng et al. (2006) model. The majority of the subpopulations are reasonably estimated, yet prison and welfare are significantly underestimated.

Our results indicate that the survey includes too few individuals who are socially close to those on welfare and in prison. Since the residuals measure this social closeness, the residuals for these two subpopulations should be rather uninformative since the people who are truly tied to these subpopulations are not in the survey. Figure 4 also indicates that unemployed people have smaller social networks, or people cannot always identify their unemployed acquaintances as such. Unemployment is also a transient status at many levels of society, making it more likely that a respondent would interact with someone who is unemployed than in the more segregated subpopulations of welfare or prison. Transmission errors (Killworth et al. 2003; 2006) may also contribute to the underrepresentation of individuals who are socially close to those in prison and on welfare. Such errors occur when a respondent knows a member of a particular subpopulation but is unaware that the person belongs to the subpopulation. Given the stigma associated with belonging to these subpopulations, individuals may be unlikely to discuss their membership with anyone besides their most trusted confidants. 


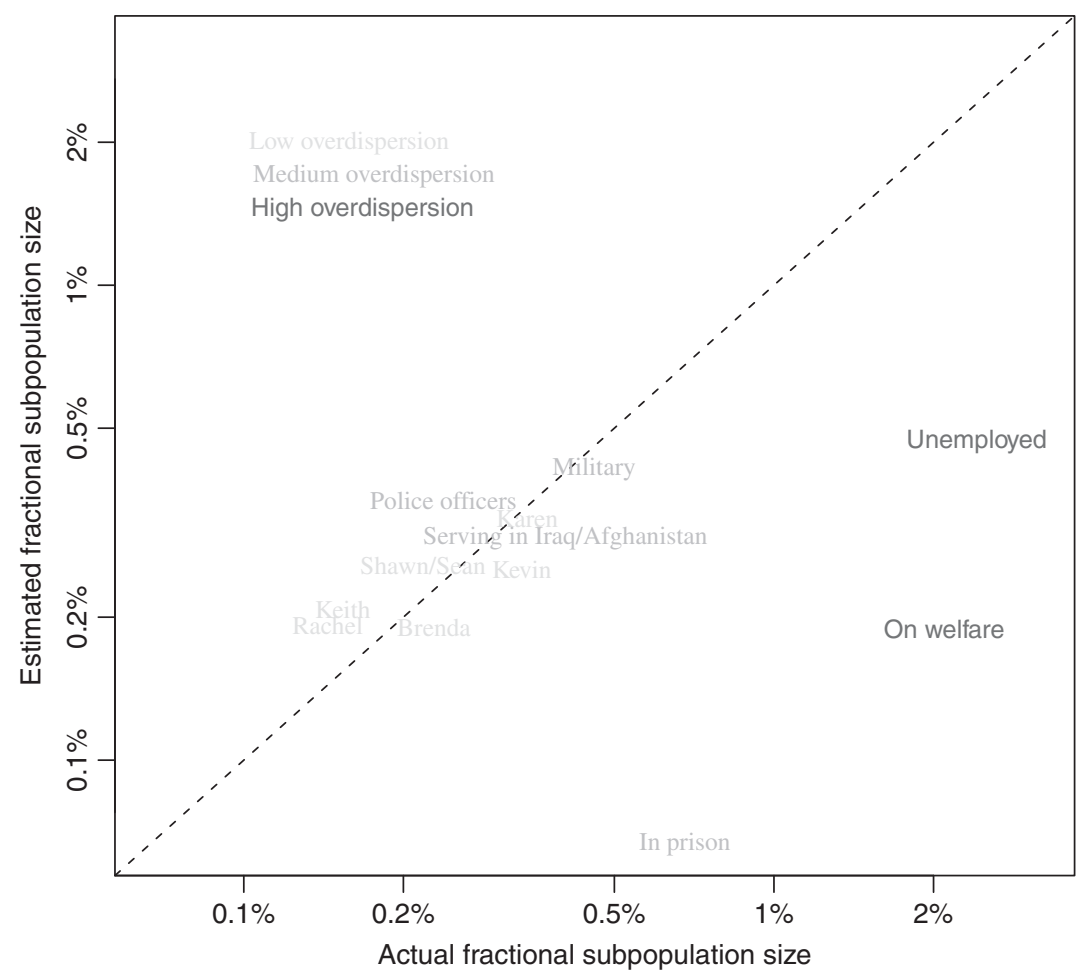

Figure 4. Comparison (on a logarithmic scale) of the estimated subpopulation sizes to their actual sizes in the United States. The figure suggests the existence of a hidden sample bias; although the sample is representative using several demographic characteristics, it includes too few individuals who are socially close to those in prison and on welfare.

\section{Discussion}

We propose the use of "How many X's do you know?" surveys (aggregated relational data, ARD) to measure the connections between individuals and subpopulations of interest. Using a continuous measure like distance rather than discrete categories to represent social structure introduces ambiguity from multiple definitions of social distance (Bottero and Prandy 2003). We have demonstrated this ambiguity in the ARD context.

The main result of our work is demonstrating that the raw counts reflect a respondent's exposure to a subpopulation while the residuals, in adjusting for degree, more reasonably represent social structure. As a template, we also consider the impact of social structure on political opinions using ARD. Both the residuals and the counts are measures of social distance, and underlying the social distance is a substantive process that could influence opinions. Being socially close to some subpopulations may influence the opinions of some respondents more than others, for example, because of the type of "social power" represented by the tie as in French and Raven (1959). Respondents who are in the military, for example, may be particularly likely to be influenced by being socially close to others in the military because they feel empathy based on their common experiences.

An additional result of our work is the discovery of latent sampling bias in our survey. The key feature of this type of bias is that it is based on the social distance of a respondent to a particular type of group and not on demographic characteristics. A potentially lucrative 
direction for future work would involve using ARD to detect hidden sampling bias. More importantly, if one could reliably estimate the bias, then a reweighting scheme could be proposed to correct for it.

In this article we have addressed how statistics derived from ARD represent information about social structure. There are also additional issues associated with the quality of ARD that we have not addressed. Respondents may know someone who is a member of a subpopulation (e.g., diabetics) but not know the person is a member of the subpopulation. We refer readers to McCormick et al. (2010) for a review of these issues and recent work to address them. Additionally, we have not focused on the mathematical properties of these estimates, but rather on the implications in the context of this scientific problem. For information about the properties of the counts under a Poisson model with overdispersion, see Zheng et al. (2006) or McCormick et al. (2010).

\section{Acknowledgments}

We thank the National Science Foundation, Columbia Population Research Center, and Columbia Applied Statistics Center for supporting this research. This work was partially completed while Tyler McCormick was supported by a Google PhD Fellowship in Statistics. We also appreciate comments by two referees and the editor.

\section{References}

Bottero, W., and K. Prandy. 2003. Social interaction distance and stratification. Br. J. Sociol., 54(2), $177-197$.

Burt, R. S. 1982. Studying status/role-sets using mass surveys. In Applied network analysis, ed. R. S. Burt and M. J. Minor, 100-118. Thousand Oaks, CA, Sage.

Burt, R. S. 1987. Social contagion and innovation: Cohesion versus structural equivalence. Am. J. Sociol., 92, 1287-1335.

DiPrete, T. A., A. Gelman, T. H. McCormick, J. Teitler, and T. Zheng. 2010. Segregation in social networks based on acquaintanceship and trust. Am. J. Sociol., 116(4), 1234-1283.

Erickson, B. H. 1988. The relational basis of attitudes. In Social structures: A network approach, ed. B. Wellman and S. D. Berkowitz, 99-121. Cambridge, UK, Cambridge University Press.

French, J. R. P., and B. H. Raven. 1959. The social basis of power. In Studies in social power, ed. D. Cartwright, 150-167. Ann Arbor, University of Michigan Press.

Hastie, T., R. Tibshirani, and J. Friedman. 2001. The elements of statistical learning. New York, Springer.

Kalmijn, M. (1994). Assortative mating by cultural and economic occupational status. Am. J. Sociol., 100(2), 422-452.

Killworth, P. D., E. C. Johnsen, C. McCarty, G. A. Shelly, and H. R. Bernard. 1998a. A social network approach to estimating seroprevalence in the United States. Social Networks, 20, 23-50.

Killworth, P. D., C. McCarty, H. R. Bernard, E. C. Johnsen, J. Domini, and G. A. Shelly. 2003. Two interpretations of reports of knowledge of subpopulation sizes. Social Networks, 25, 141-160.

Killworth, P. D., C. McCarty, H. R. Bernard, G. A. Shelly, and E. C. Johnsen. 1998b. Estimation of seroprevalence, rape, and homelessness in the U.S. using a social network approach. Eval. Rev., 22, 289-308.

Killworth, P. D., C. McCarty, E. C. Johnsen, H. R. Bernard, and G. A. Shelley. 2006. Investigating the variation of personal network size under unknown error conditions. Sociol. Methods Res., $35(1), 84-112$.

McCormick, T. H., A. Moussa, J. Ruf, T. A. DiPrete, A. Gelman, J. Teitler, and T. Zheng. 2009. Comparing two methods for predicting opinions using social structure. In Proceedings of the Joint Statistical Meetings, August, Washington, DC, American Statistical Association. 
McCormick, T. H., M. J. Salganik, and T. Zheng. 2010. How many people do you know?: Efficiently estimating personal network size. J. Am. Stat. Assoc., 105(489), 59-70.

MIT Web. 2007. CCES. http://web.mit.edu/polisci/portl/cces/index.html

Moscovici, S. 1985. Social inuence and conformity. In Lindzey, G. and Aronson, E., editors, The Handbook of Social Psychology, volume 2, pages 347-412. Random House.

Newman, M. E. J. 2003. The structure and function of complex networks. SIAM Rev., 45, $167-256$.

Venables, W. N., and B. D. Ripley. 2002. Modern applied statistics with S. New York, Springer.

Zheng, T., M. J. Salganik, and A. Gelman. 2006. How many people do you know in prison?: Using overdispersion in count data to estimate social structure. J. Am. Stat. Assoc., 101, 409-423. 